\title{
Poïétique multi-paysagère, quand l'œuvre fait système avec son environnement
}

\author{
Multi-landscape poetics, when artwork makes a system with its
} environment

\author{
Raphaël Bergère ${ }^{1}$, Mathilde Murat ${ }^{1}$ \\ ${ }^{1}$ Artistes et docteurs en Arts plastiques, Chercheurs associés au LARA-SEPPIA, Université Toulouse - Jean Jaurès
}

RÉSUMÉ. La démarche artistique que nous présentons dans cet article s'inscrit dans une perspective paysagère que nous analyserons selon une approche complexe et écosystémique.

L'œuvre dont il est question, intitulée Luminale, actualise la figure de la cabane pour interroger nos rapports aux environnement et aux technologies, tout en se construisant à travers une multiplicité de paysages.

En référence à des philosophes qui prônent une vison modeste et poétique de l'habitat (Thoreau, Bachelard), nous étudions dans un premier temps en quoi cette figure de la cabane nous permet d'interroger l'habitat et la technique dans une dimension nomade.

Dans un second temps, le présent article s'intéresse davantage à l'aspect sauvage qui se dégage de l'installation Luminale. En nous appuyant sur le travail de théoriciens ayant abordé des questions de dispositifs interactif (Couvhot), d'espace plastique (Guerin) et notamment la notion de figural (Deleuze) nous verrons comment la scénographie interactive de Luminale immerge les spectateurs dans un écosystème artistique singulier. L'expérience de l'œuvre permet en effet de revisiter les paysages visuels et sonores à partir desquelles elle a été constituée, nous invitant alors à ralentir pour prendre le temps d'observer et d'écouter l'environnement.

ABSTRACT. In this article we are going to present an artistic process within a landscape perspective, which scopes with a complex an ecosystemic approach.

Our art project named Luminale was made while exploring a multiplicity of environment. Within this artwork, we want to update the symbolic of the "cabin" to question our relationships to the environment and technologies.

With reference to philosophers who advocate a modest and poetic vision of the habitat (Thoreau, Bachelard), we first study how the symbolic of the "cabin" allows us to question the habitat and technologies in a nomadic way.

Secondly, this article focuses more on the wilderness dimension existing in Luminale art installation. Invoking the work of theorists who have addressed questions of interactive devices (Couchot), plastic space (Guerin) and in particular the notion of "figural" (Deleuze), we will demonstrate furthermore how Luminale's interactive scenography immerses spectators in a unique artistic ecosystem. The work's experience allows us to revisit the visual and sound landscapes from which it was composed, inviting us to slow down and take the time to observe and listen to the environment around us.

MOTS-CLÉS. Art, environnement, paysage, nomadisme, sauvage, dispositif, scénographie, interactivité.

KEYWORDS. Art, environment, landscape, nomadism, wild, device, scenography, interactivity.

Cet acte fait suite à une communication que nous avons réalisée en binôme. Ce choix de communication à deux voix nous semblait évident pour parler d'une œuvre que nous avons coréalisée.

Intitulée Luminale, l'œuvre en question est pensée comme un processus à partir duquel ont émergé plusieurs productions artistiques liées les unes aux autres.

Luminale, c'est une installation qui tisse des liens particuliers et significatifs avec différents environnements : celui où le matériel a été trouvé et récupéré, celui où elle a été construite, et ceux dans lesquels elle a été installée puis exposée.

Plus précisément, cette démarche nous a menés à la production d'une cabane lumineuse, qui a ensuite voyagé pour faire l'objet d'accrochages dans quatre environnements différents, avant de 
faire son retour sous forme d'installation interactive dans un environnement d'exposition. Une série de tirages photographiques sur bois, présente en contrepoint de l'installation, des vues de la cabane dans chacun des environnements.
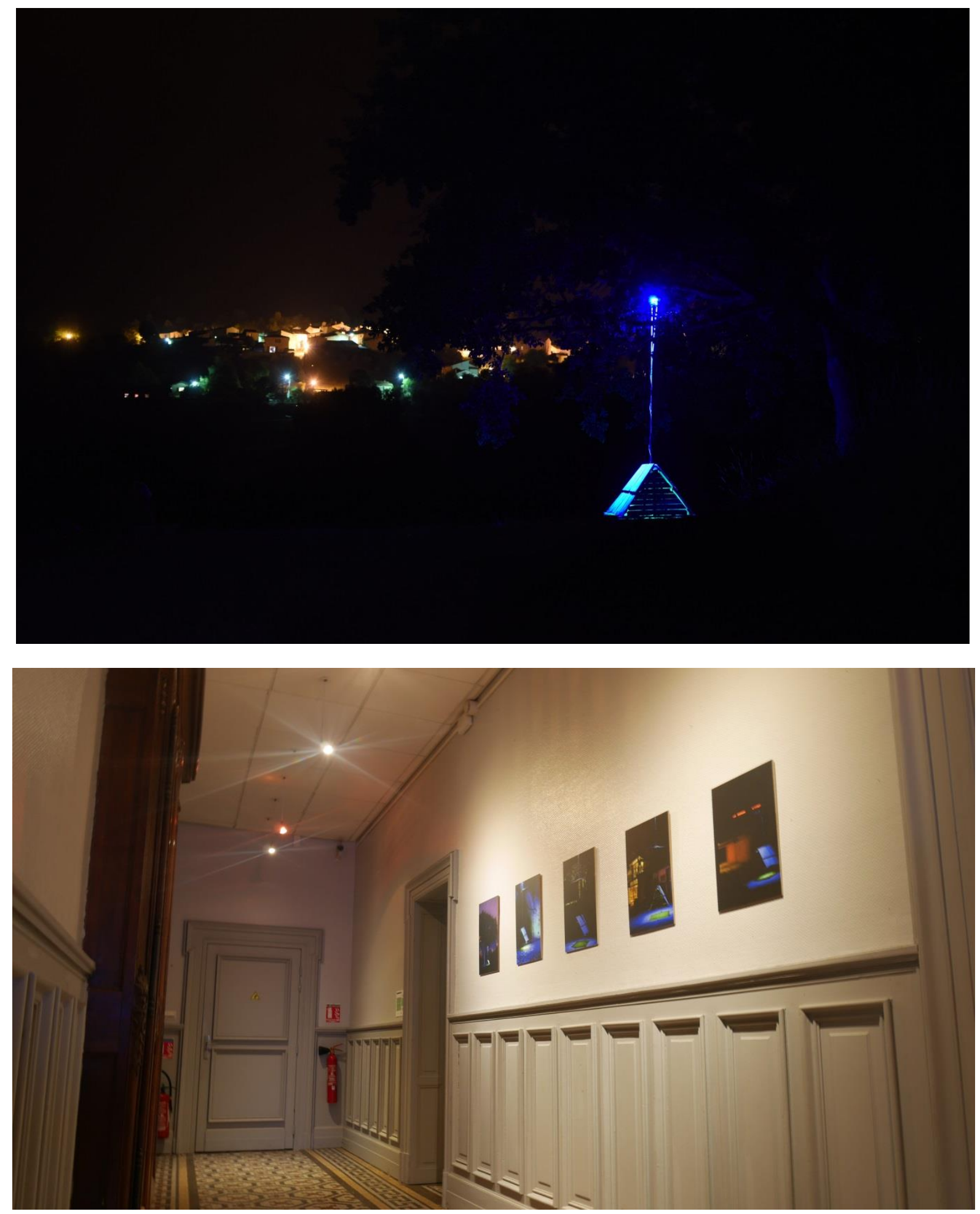

L'installation a été conçue pour être suspendue à une corde et flotter à environ cinquante centimètres du sol. D'environ un mètre carré de superficie, la cabane n'est donc pas habitable à proprement parler, mais elle fait l'objet d'un travail et d'une recherche esthétique autour de la lumière et du paysage. Une lumière verte est diffusée à l'intérieur, elle est visible à travers les interstices des planches, mais elle se diffuse aussi sur le sol au-dessous de la cabane. Cette dernière n'ayant pas de plancher, elle est donc d'autant plus inhabitable. Un autre projecteur lumineux fixé en hauteur à l'extérieur de la cabane diffuse une lumière bleue sur la paroi extérieure de la cabane et 
notamment dans l'environnement alentour. Bien que cette cabane ne soit pas habitable, elle semble par contre elle-même habiter son environnement.

La question du paysage sera donc étudiée au regard de ce projet artistique caractérisé par sa multiplicité : multiplicité de matériaux, de techniques, d'environnements de création et de modes de monstration au public. Mais surtout, nous souhaitons étudier Luminale en tant qu'œuvre constituée à partir des multiples paysages qu'elle a traversés et dont elle garde la trace. Il est important de préciser qu'il s'agit autant de paysage sonore que de paysage photographique. C'est donc une démarche qui s'inscrit à la croisée de l'installation, du paysage sonore et de la photographie paysagère, mais aussi des arts technologiques.

Aborder le paysage par le biais d'une installation technologique (capteurs, dispositif sonore, éclairage électroluminescent) est une façon pour nous de questionner nos rapports aux technologies et aux environnements, notamment d'un point de vue écosystémique.

Si nous partons du postulat qu'habiter, c'est faire système avec son environnement, nous inscrirons notre analyse de cette installation dans la perspective d'une pensée paysagère écosystémique. Nous verrons alors dans un premier temps que Luminale relève d'une poïétique paysagère selon une approche nomade de sa relation aux écosystèmes. Dans un second temps, l'œuvre sera étudiée dans une perspective écosystémique qui confère à l'œuvre un aspect sauvage ou animal, en nous appuyant sur la notion de figural (Deleuze).

\section{Installation nomade et paysages multiples : donner forme à l'objet paysager}

Luminale est née d'une volonté de mener une pratique artistique en croisant des matériaux comme le bois, la terre et la lumière, afin de réaliser cette cabane lumineuse et sonore. La cabane, c'est typiquement l'économie de moyens, le bricolage et l'habitat construit soi-même, souvent avec du matériel récupéré et trouvé dans l'environnement où elle est construite. La cabane est aussi symbole de liberté, qu'il s'agisse de liberté individuelle comme de liberté créative. Habiter dans une cabane au milieu des bois (même temporairement), c'est aussi une expérience d'émancipation par rapports à des systèmes urbains et sociaux. C'est bien ce que Henri-David Thoreau évoquait dans Walden ou la vie dans les bois ${ }^{1}$. Nous citons aussi le philosophe Gaston Bachelard qui dans la Poétique de l'espace ${ }^{2}$ aborde pour sa part la question de l'habitat modeste d'un point de vue poétique. Il y compare par exemple l'âme humaine avec la maison et ses différentes pièces. Il évoque même la hutte, en faisant l'éloge de sa simplicité : "La hutte ne peut recevoir aucune richesse "de ce monde ». Elle a une heureuse intensité de pauvreté. La hutte de l'ermite est une gloire de la pauvreté. De dépouillement en dépouillement, elle nous donne accès à l'absolu du refuge. $\gg^{3}$

Luminale a été construite à partir de bois trouvé proche de la maison d'enfance de Mathilde. Le choix d'utiliser des matériaux trouvés dans cet environnement n'est pas anodin. Nous avons en effet cherché du bois usé par le temps et l'humidité, matériau qui porte en lui les marques du temps passé dans cet environnement. Pour trouver les planches qui permettraient de construire la cabane, nous

\footnotetext{
${ }^{1}$ Henry David Thoreau, Walden ou La vie dans les bois, Paris : éd. Climats, 1967, [traduction et notes par Germaine LandréAugier].

${ }^{2}$ Gaston Bachelard, La poétique de l'espace, Paris, Presses universitaires de France, 2012.

${ }^{3}$ Gaston Bachelard, ibid, p 46.
} 
avons arpenté les alentours de la maison, mais aussi des forêts dans les environs à la recherche de planches et autres cabanes abandonnées pouvant nourrir notre processus créatif.

Il est important de préciser que notre approche de la cabane n'est pas tournée vers une vision romantique, pas non plus vers l'éloge d'un archaïsme teinté de retour à la nature. Ce projet était en effet une occasion de réinterroger l'enfance, mais aussi de confronter les temps passés avec les temps à venir.

C'est aussi une œuvre qui se caractérise par le fait que nous avons tous les deux grandi à la campagne, mais aussi grandi avec des esthétiques fluo et autres lumières acidulées des années 80 et 90, avec des séries comme X-Files et autres films de science fictions qui mettent en scène de nouvelles technologies et des esthétiques baignées de lumières saturées.

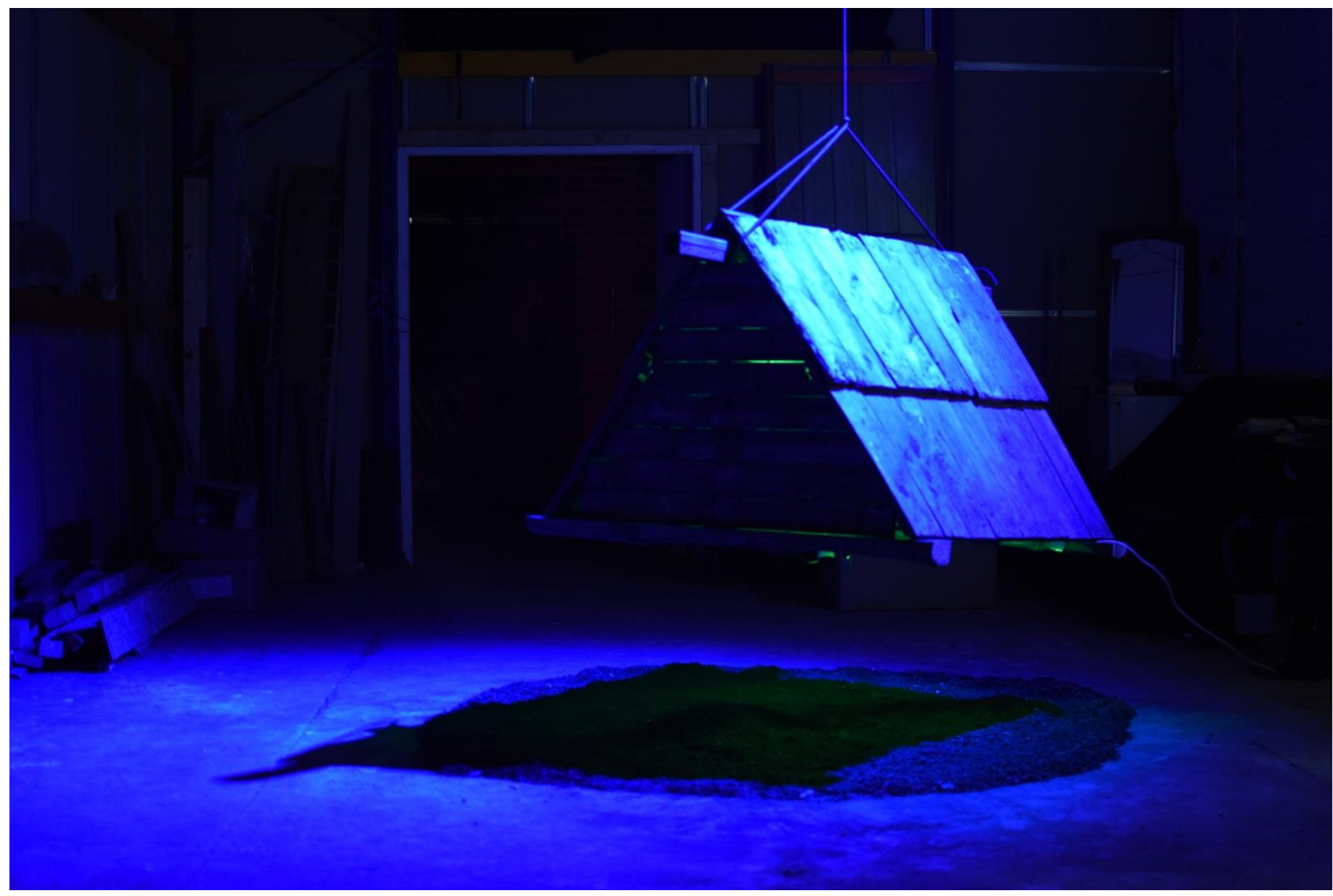

Une fois les matériaux collectés, la cabane a été construite dans notre atelier à Mix'Art Myrys ${ }^{4}$ à Toulouse. Avant de la présenter au public dans un environnement d'exposition, nous avons décidé de l'installer dans différents environnements qui n'ont rien à voir avec des lieux d'expositions et qui, au contraire, se situent même parfois loin des regards.

Des accrochages ont donc eu lieu dans quatre environnements.

Le premier était un tunnel sous le périphérique toulousain.

\footnotetext{
${ }^{4}$ Collectif d'artistes mettant à disposition des espaces de création.
} 

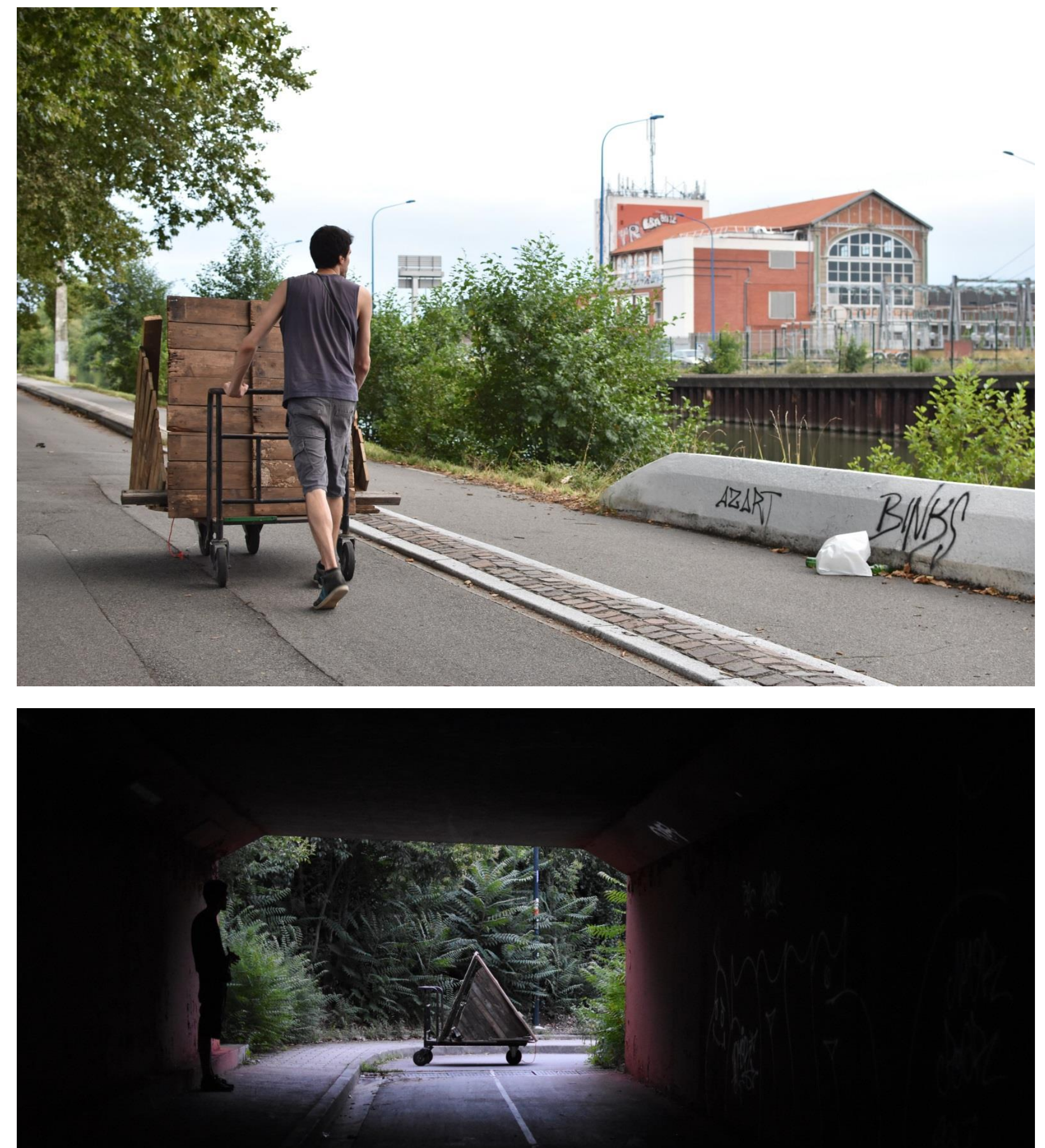

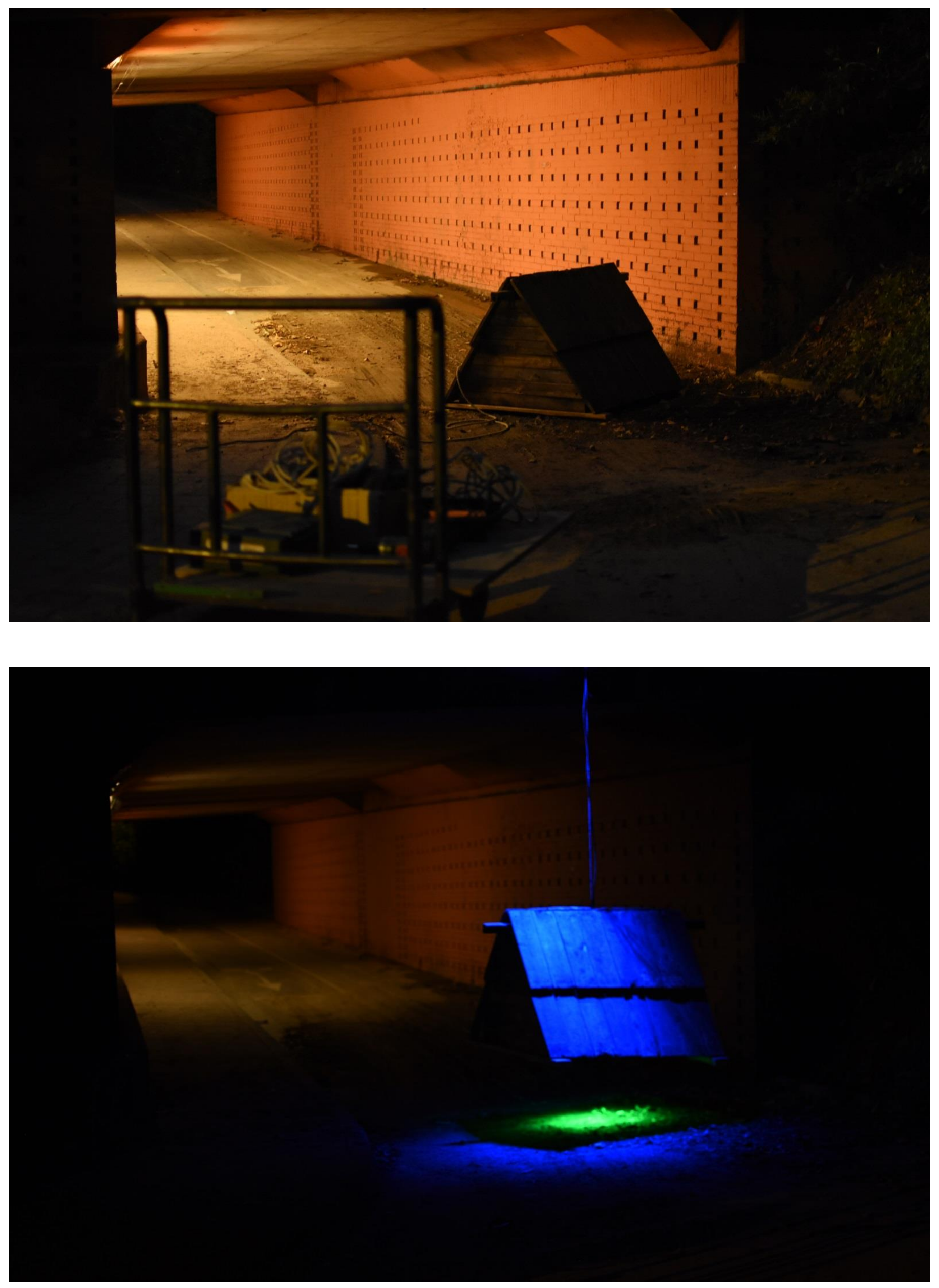
En fin de journée, nous avons déplacé tout le matériel de la cabane en dehors de l'atelier, pour la remonter et la suspendre sous le périphérique. La cabane était installée avec sa mise en lumière particulière : une lampe verte à l'intérieur de la cabane et une bleue à l'extérieur, qui diffusaient une lueur artificielle saturée et presque acidulée.

Cette installation était l'occasion de faire dialoguer la cabane et sa lumière, avec les autres lumières du tunnel et celles des voitures qui circulaient rapidement sur le périphérique, mais aussi avec le paysage sonore : bruits de voitures, passants à vélos, un scooter qui passait sous le tunnel.

Le second environnement était une ancienne usine en bordure de village, reconvertie en résidence d'artistes associative, à Axat dans les Pyrénées du département de l'Aude.

Nous avons ici suspendu la cabane à une barre en fer qui traversait la partie de l'usine aux allures de grange, pour capturer à deux reprises l'intensité de l'instant : une première fois à la tombée de la nuit, et une autre le lendemain à l'aube. Autour des lumières vertes et bleues de la cabane, il y avait une lumière chaude qui donnait une teinte orangée au bois de la grange et au matériel stocké un peu partout à l'intérieur. Le lendemain à l'aube, la lumière du matin apparaissait à travers les interstices des planches.

Nous avons aussi capté des paysages sonores de cet environnement. Quelques véhicules qui passaient sur la route à proximité, des bruits de machines au travail, une scie, la pluie qui s'est abattue un moment sur le toit du bâtiment. A l'aube, le village donnait ses premiers signes d'éveil : des véhicules qui partent au travail, un chien qui aboie, un coq qui chante... 


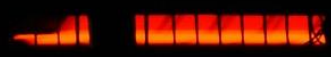

iseyesenge

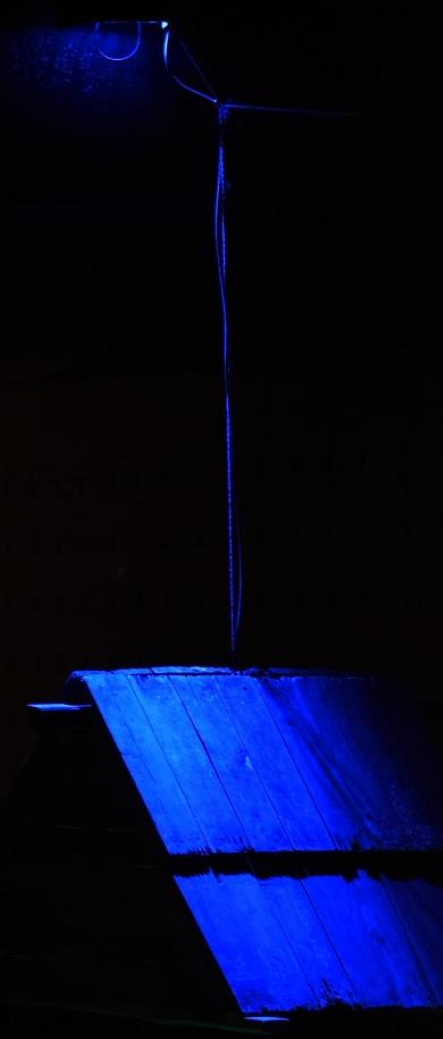

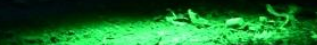

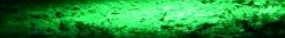




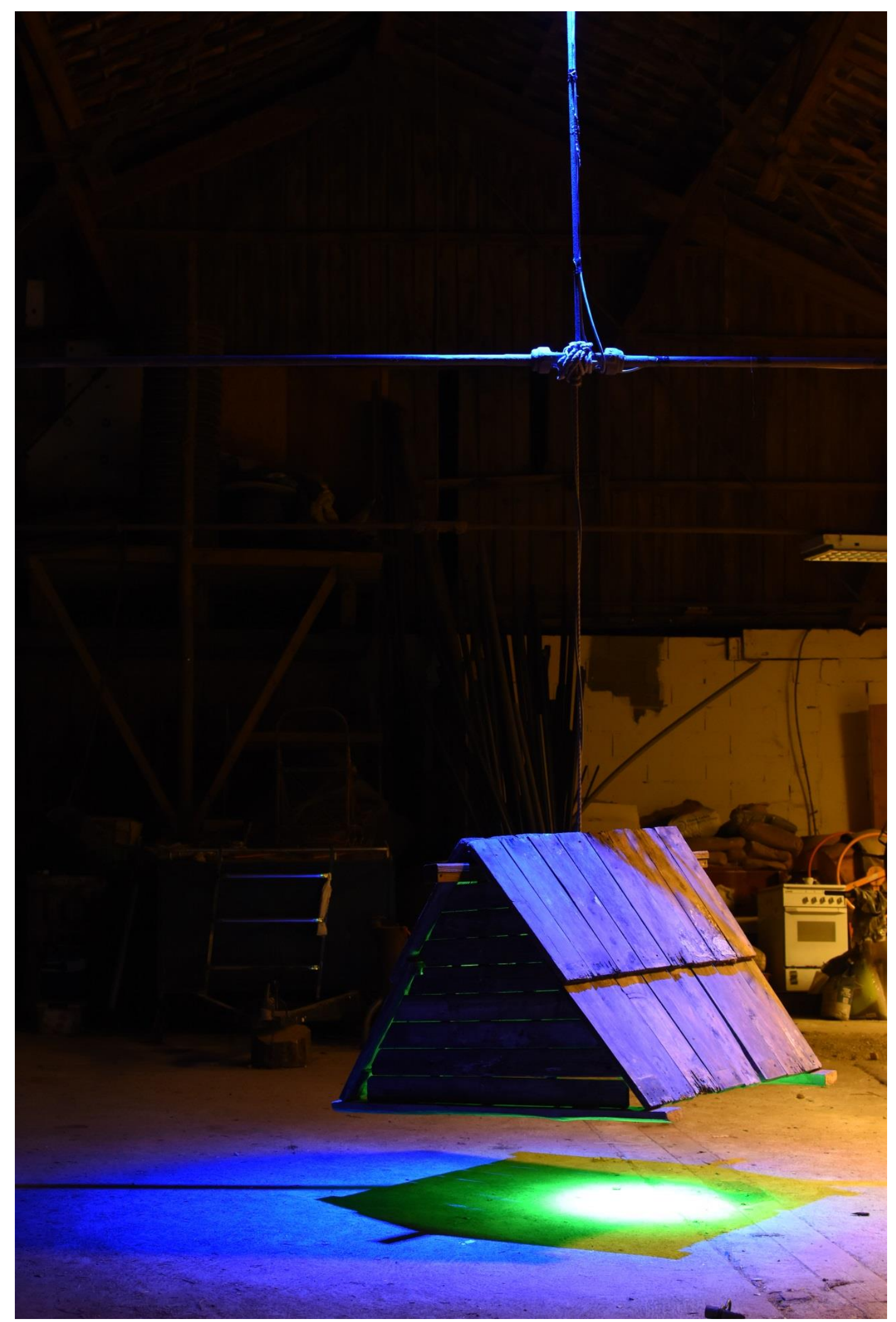




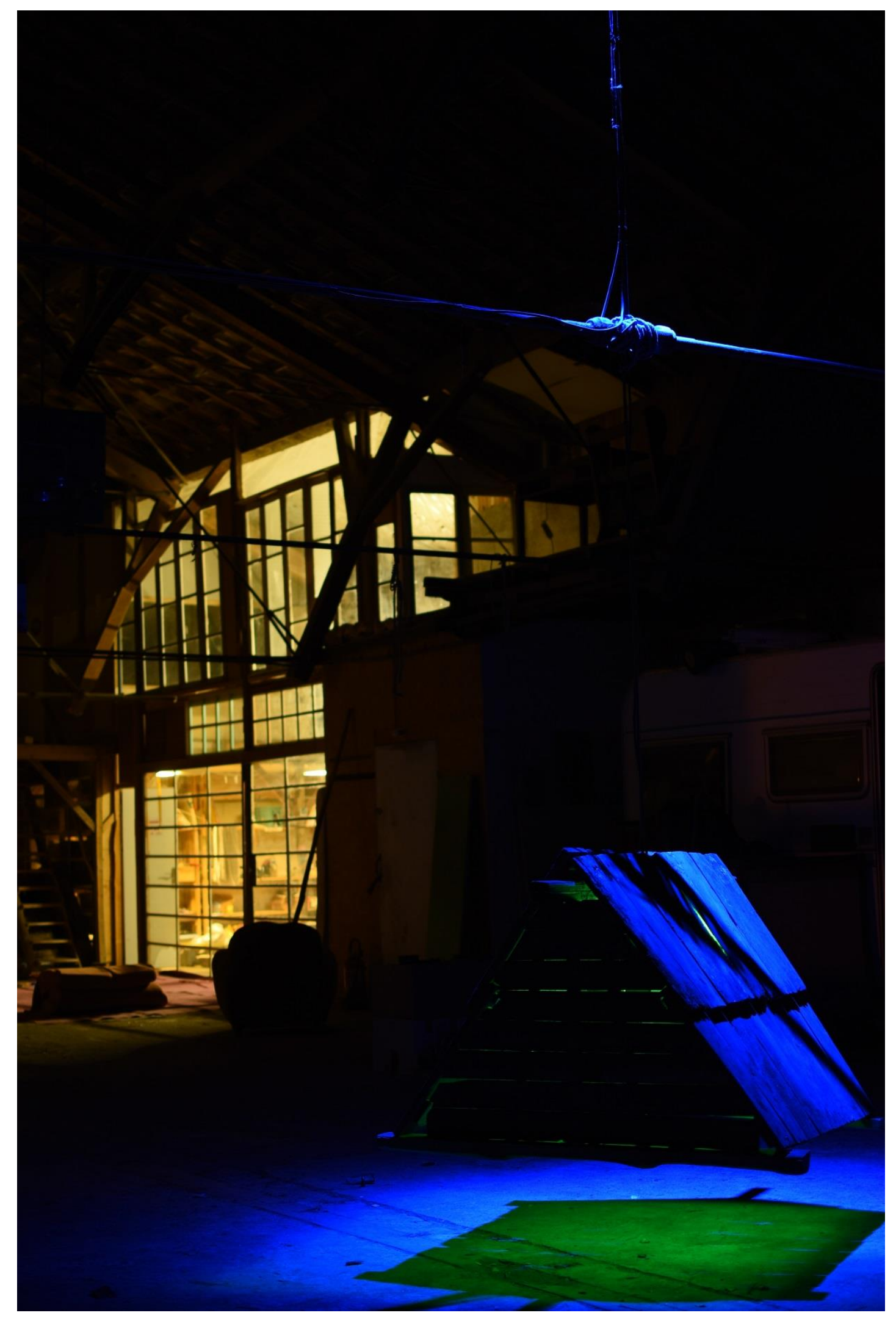




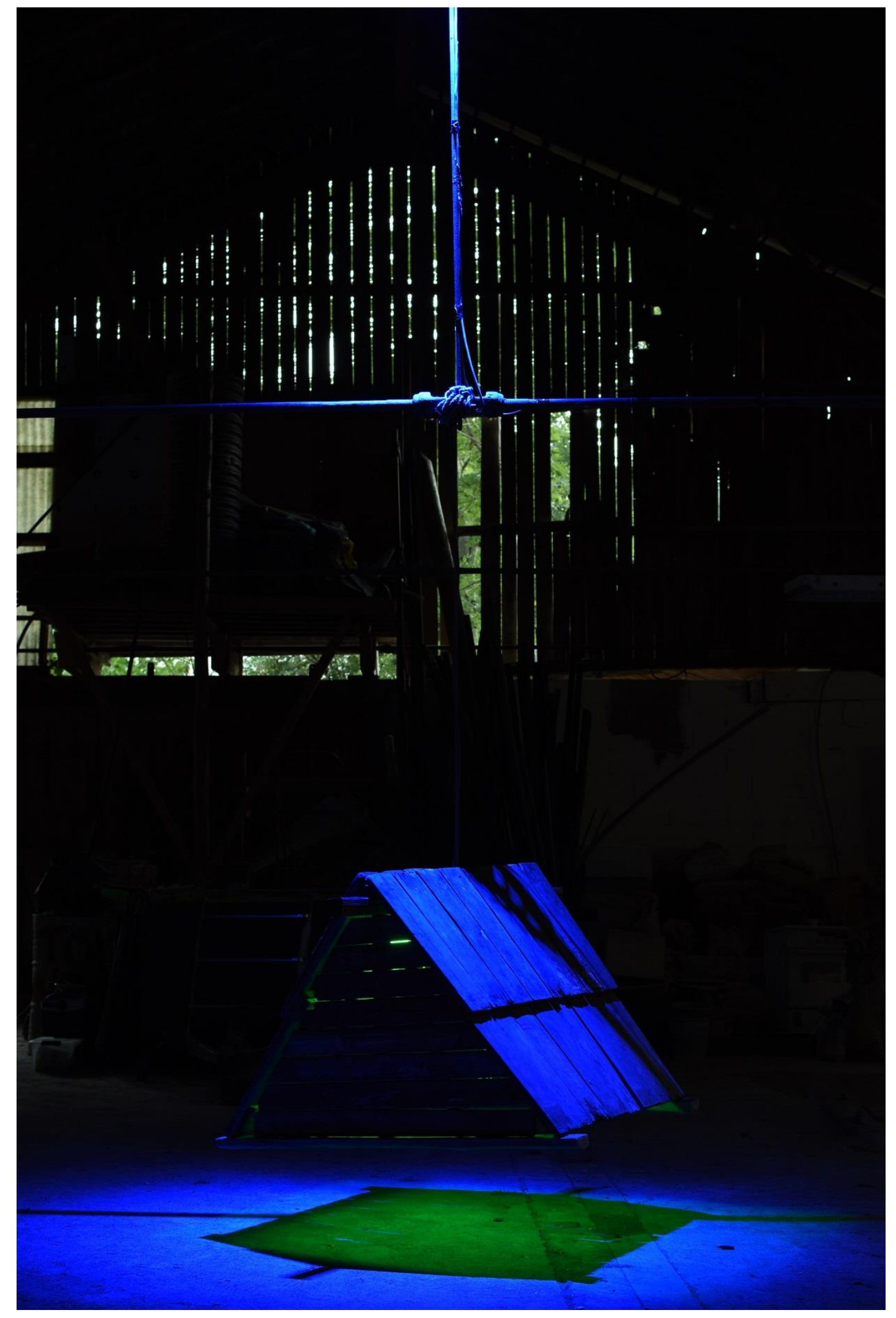


Le troisième environnement était un tunnel en bordure de village sous lequel passait un ruisseau venant de la forêt. Ce tunnel nous a paru assez fascinant car il marquait vraiment la limite entre l'environnement façonné du village et l'environnement de forêt assez dense et non entretenu duquel provenait le ruisseau.

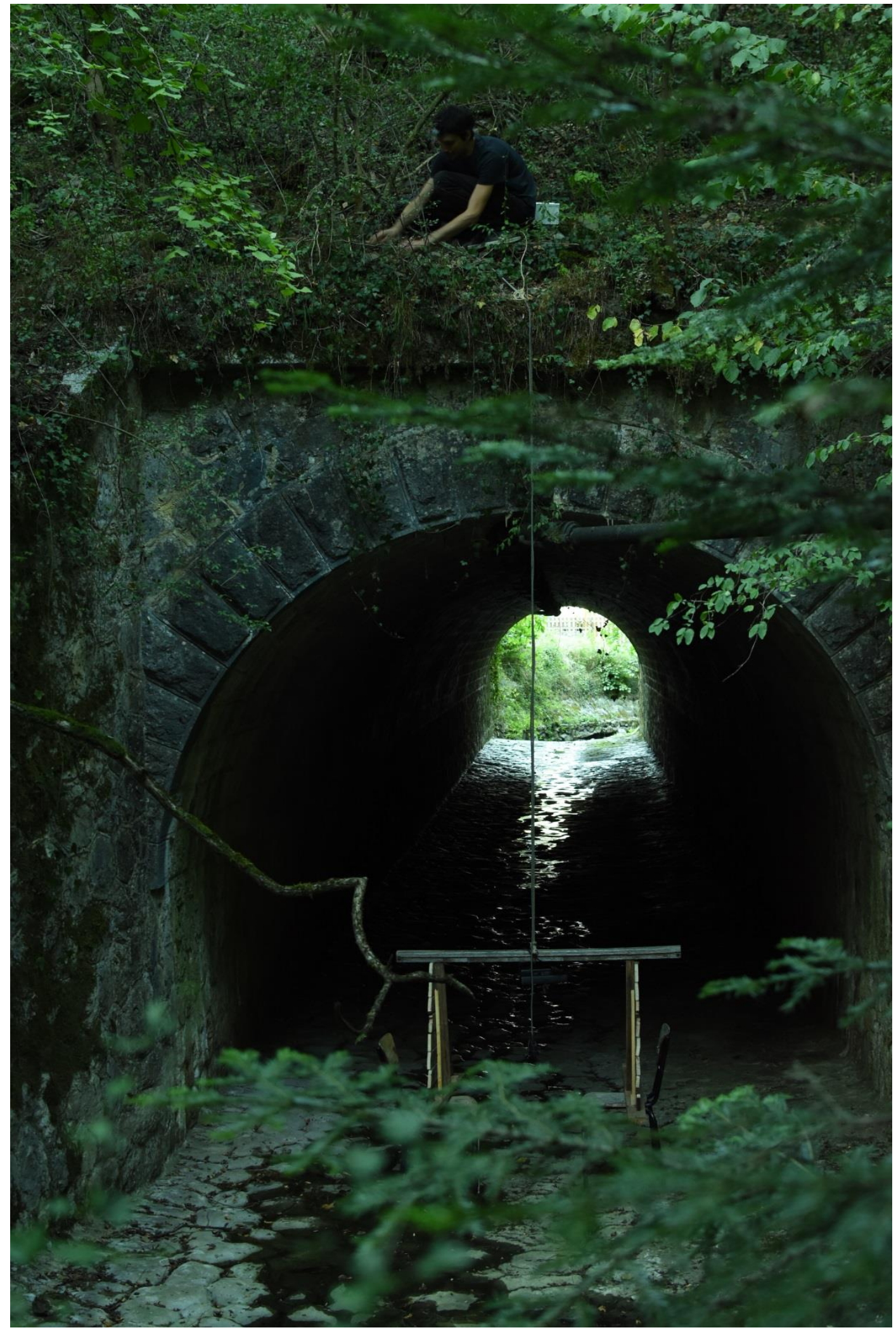


Les photographiées réalisées rendent compte de la lumière bleutée qui se répandait sur les pierres du tunnel, puis de la cabane suspendue et sa lumière reflétée sur les galets et l'eau du ruisseau.

Ce moment nous a particulièrement marqué, autant d'un point de vue visuel que sonore. Les reflets dans l'eau et sur la paroi du tunnel étaient accompagnés du léger bruit du ruissellement, et des bruits de nos pas qui faisaient échos sous le tunnel.

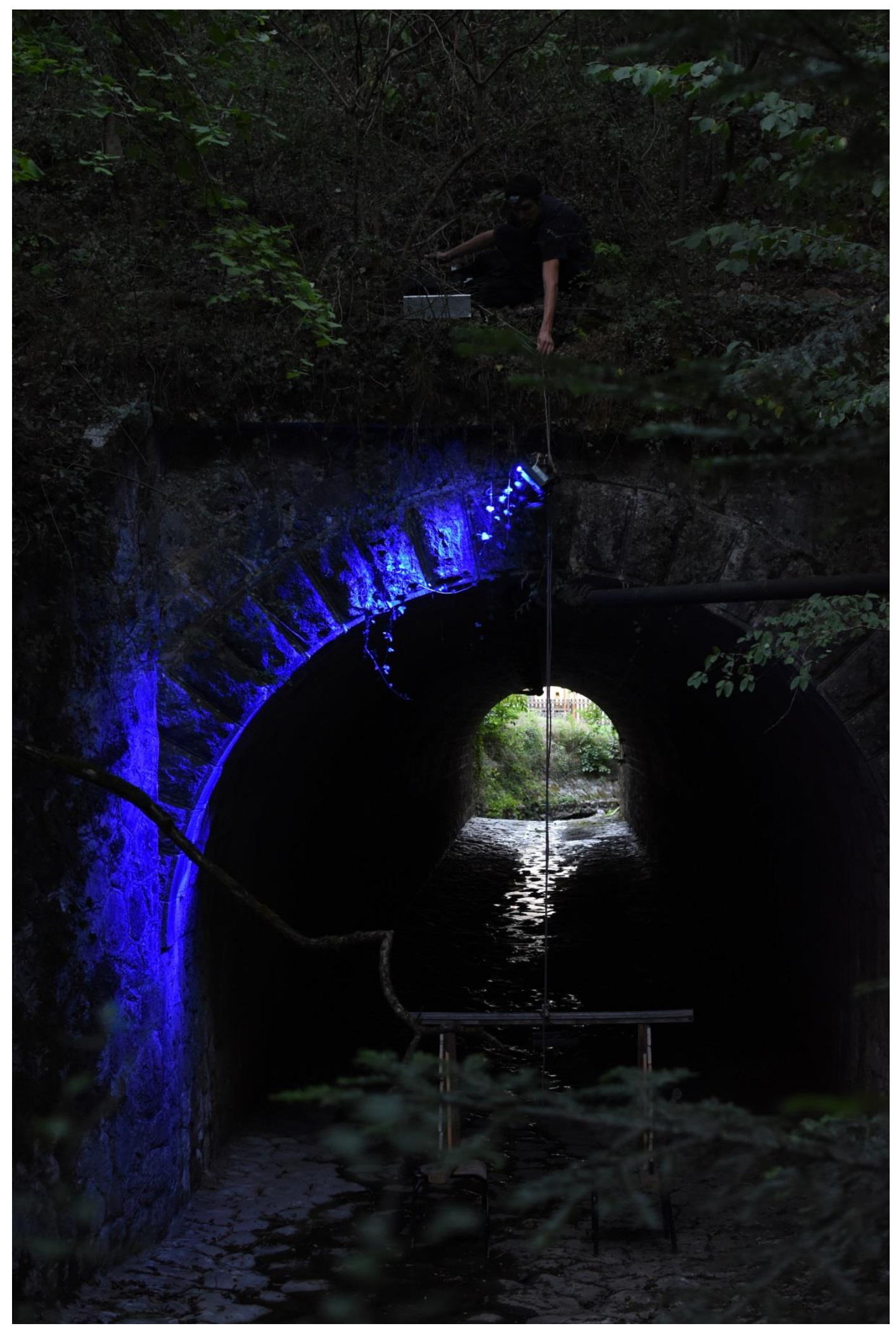




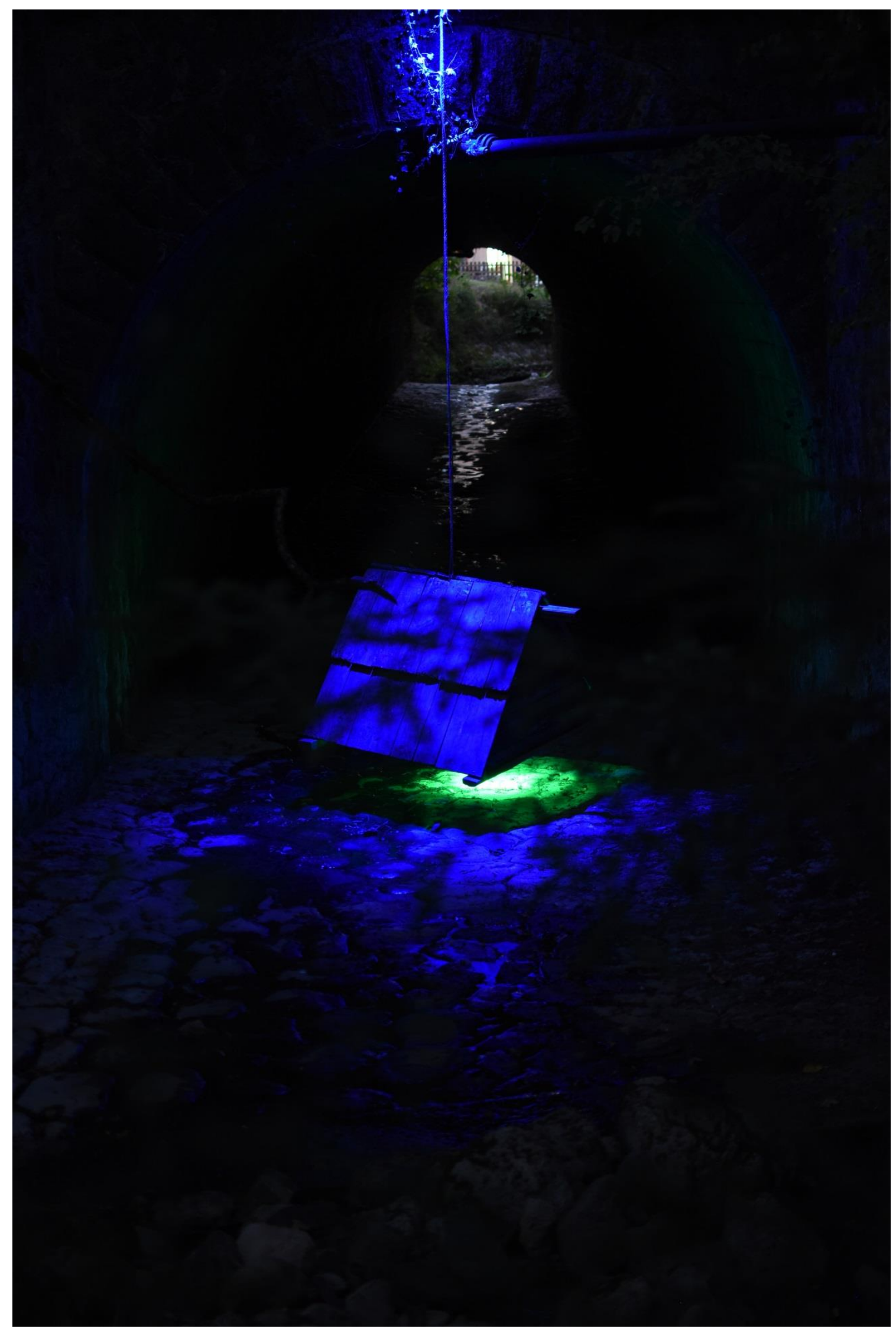




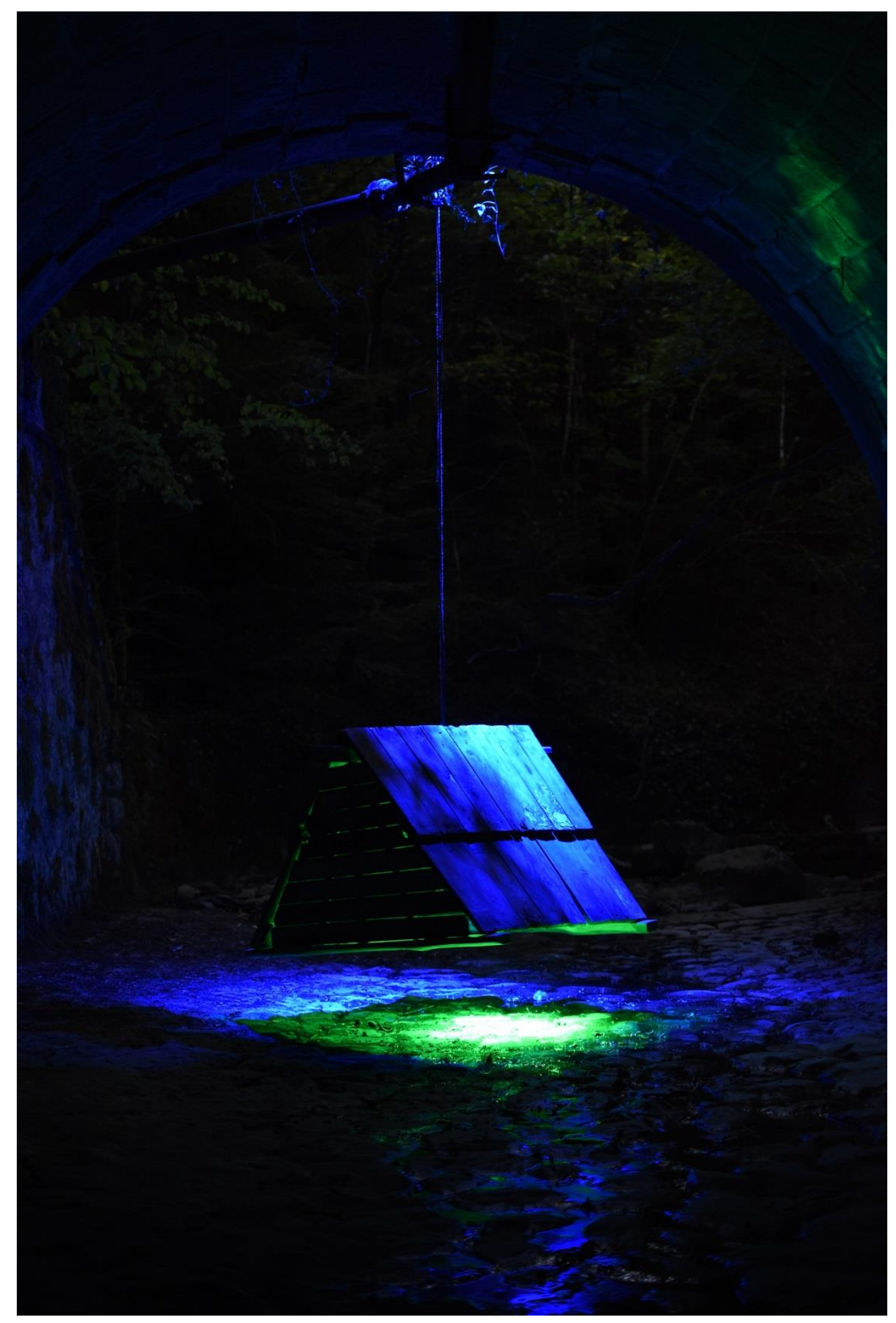




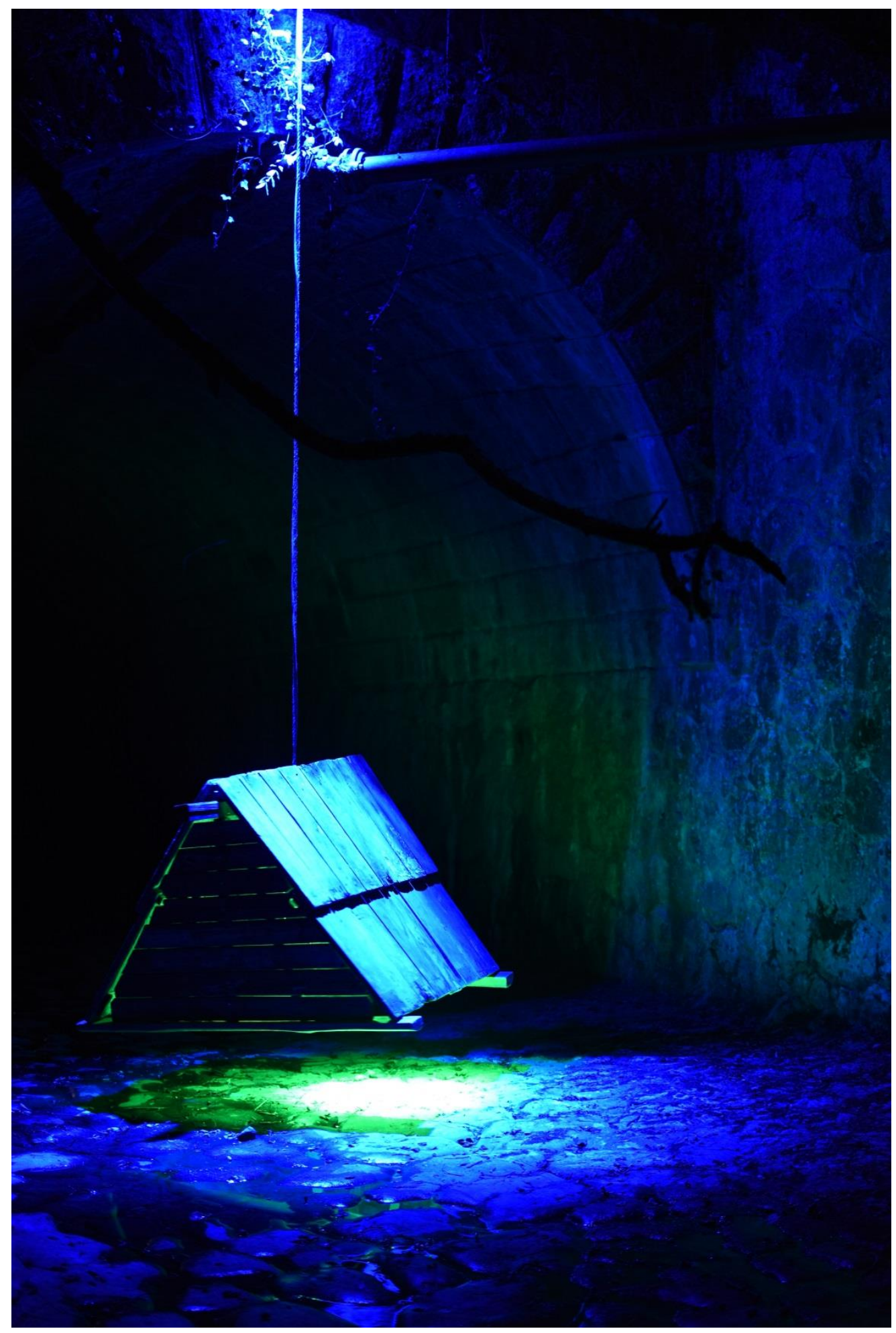


Enfin, le quatrième environnement se trouvait en haut d'une colline, en face d'un petit hameau de montagne.

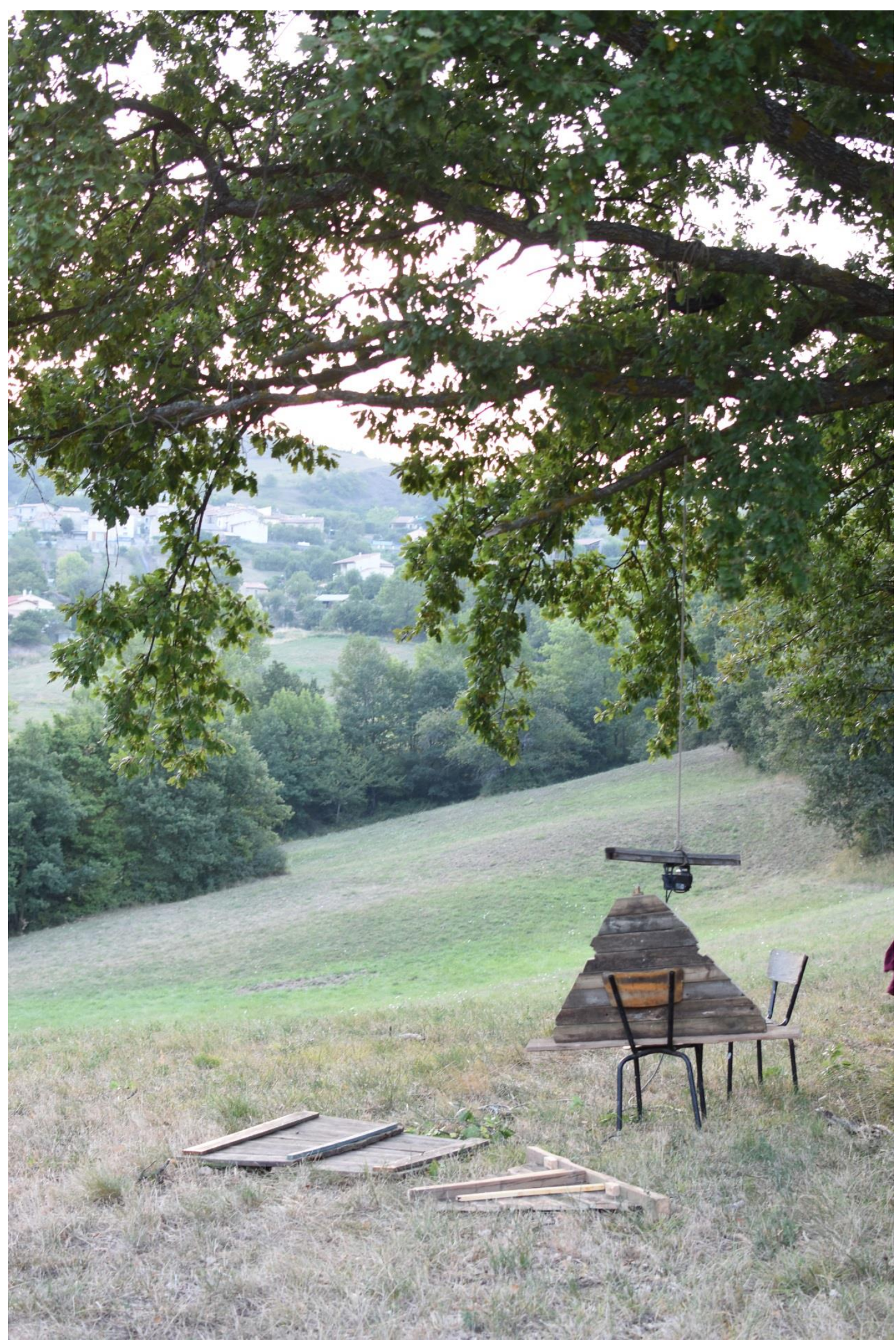




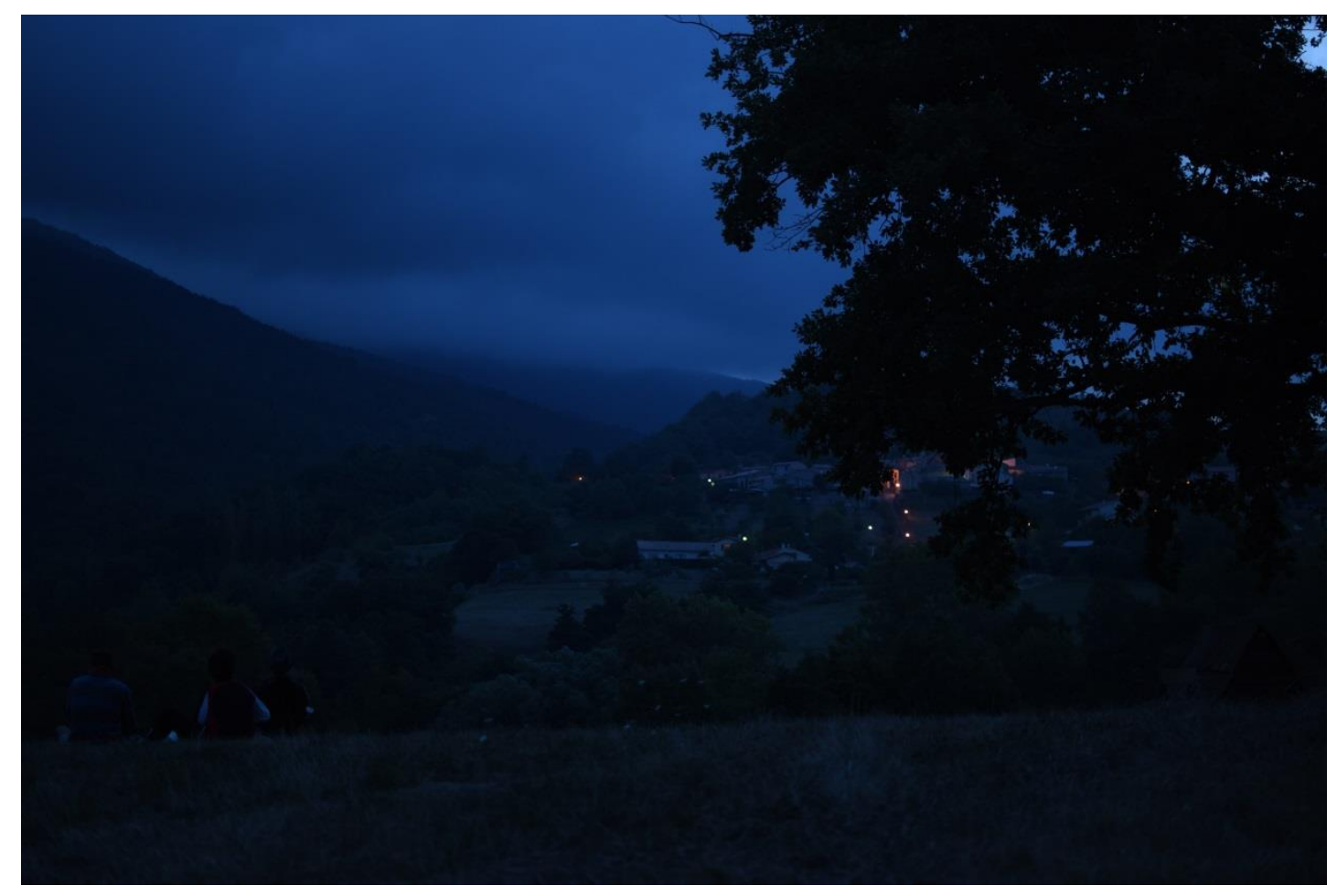

Ici, la cabane était accrochée sous un grand arbre. A la tombée de la nuit, les lumières de la cabane ont croisé celles du ciel étoilé, mais aussi celles du village pris dans la brume qui montait dans la vallée. Nous avons réalisé des prises de son des grillons, parfois d'un chien qui aboyait au loin et d'autres bruits du village sur l'autre versant de la colline.

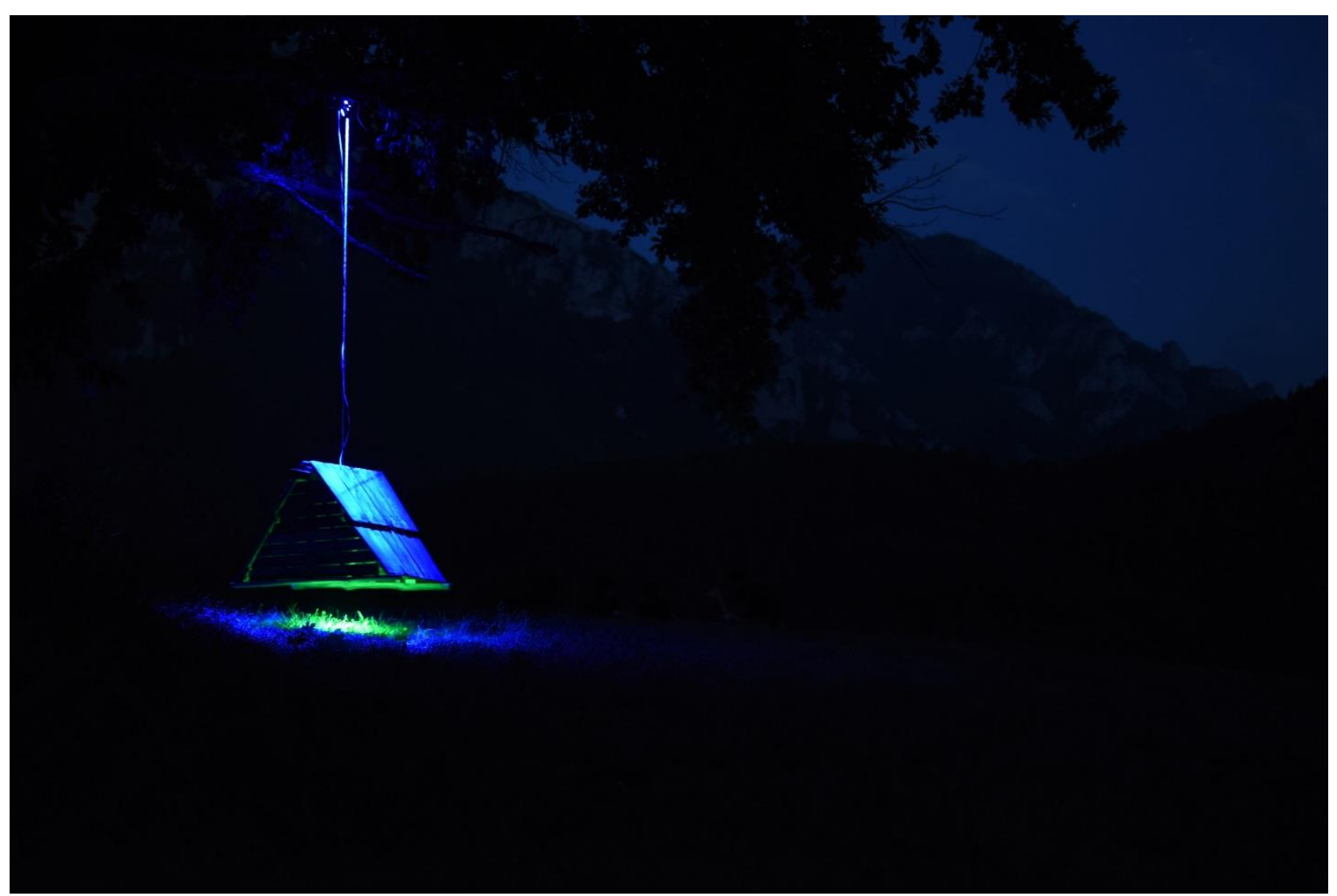



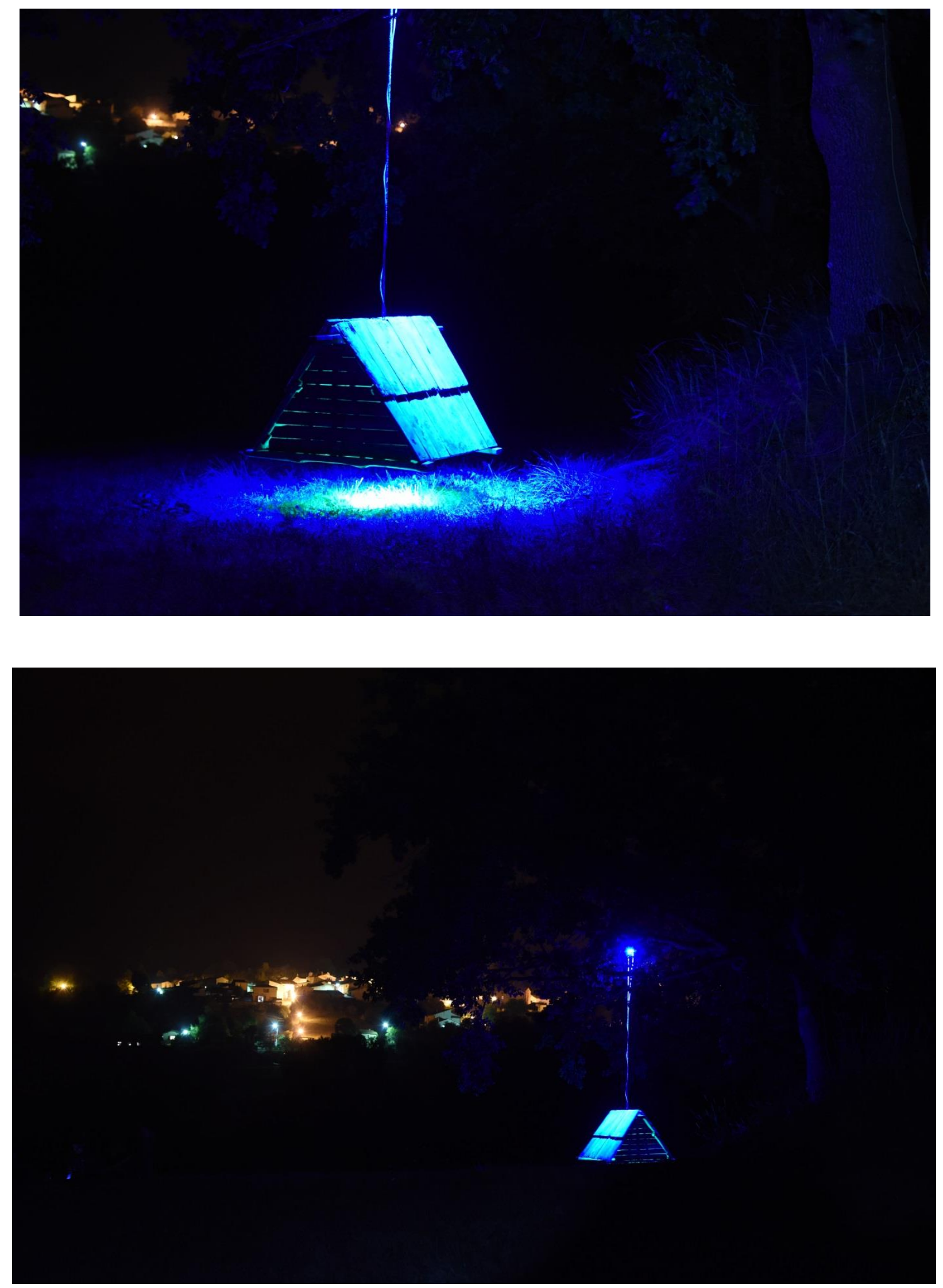


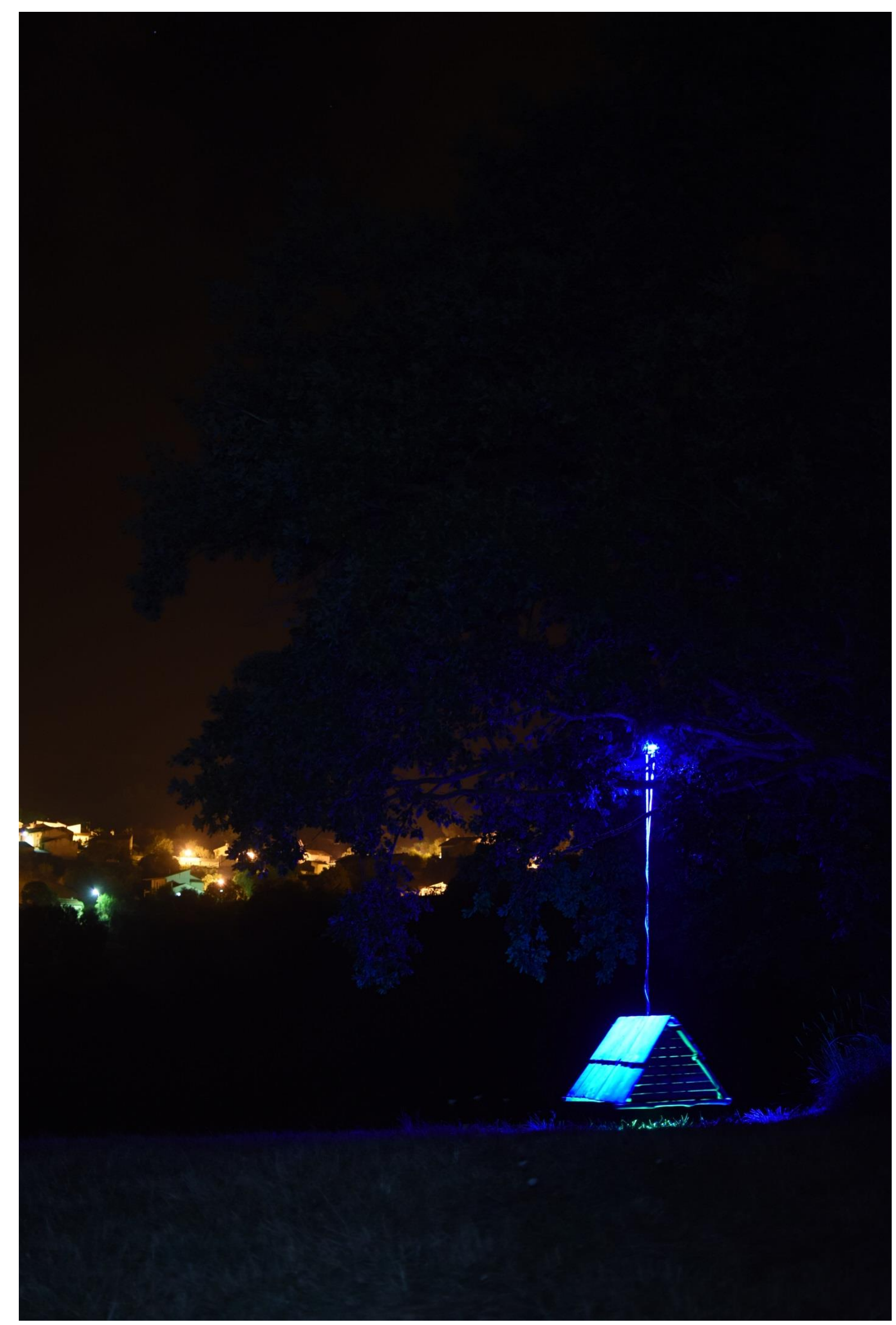


Toute cette démarche relève pour nous d'une poïétique multi-paysagère. L'œuvre est en effet très fortement caractérisée par nos expériences d'immersion et de reconfiguration des paysages rencontrés. Nous envisageons ici le paysage comme une composition visuelle et sonore liée à nos expériences sensibles dans ces différents environnements. En dehors des raisons pratiques (nécessité de points d'accroches en hauteur) qui ont joué un rôle dans le choix de ces environnements, nous les avons surtout choisis pour leur richesse sonore et visuelle. Luminale avait été pensée pour entrer en dialogue avec un environnement et ainsi faire paysage. En ce sens, elle interroge nos façons d'habiter technologiquement et artistiquement le monde.

L'ensemble de ces expériences forme alors un paysage multiple et imprégné de complexité, où des traces de constructions anciennes côtoient le caractère éphémère des lumières artificielles branchées sur une batterie. Ce sont des paysages qui intègrent de la rapidité, celle par exemple des véhicules, mais qui intègrent aussi de l'apaisement, celui du ruissellement lent et aiguë de l'eau au milieu des pierres, ou celui des grillons.

Luminale est un objet artistique nomade et électrique emmené dans des lieux qui ne sont pas reliés à un réseau électrique et encore moins prévus pour accueillir des œuvres. La question du nomadisme associé aux objets techniques est étroitement liée à nos expériences d'être au monde. Les technologies, à commencer par celles du déplacement (véhicules, chemins de fers, voyage aérien...) permettent de se déplacer beaucoup plus aisément sur le globe. Désormais, les smartphones équipés de GPS permettent de nous repérer très facilement, d'organiser nos déplacements et de trouver très rapidement une grande quantité d'informations sur les lieux que nous visitons.

Mais cette mobilité semble avoir un coût. Qu'il s'agisse de nos déplacements, des déplacements des objets techniques eux-mêmes (souvent fabriqués sur un continent pour être vendus sur un autre) ou du déplacement incessant de flux d'informations, le problème du coût écologique de cette mobilité prend de plus en plus d'importance au niveau mondial, à un tel point que l'utilisation de certains de ces objets techniques devient culpabilisante.

Il y a dans Luminale une volonté de se confronter à ces contradictions, qui sont selon nous caractéristiques d'une génération fascinée par le potentiel artistique des technologies tout en étant consciente des problèmes écologiques planétaires.

Pour autant, le nomadisme de Luminale traduit pour nous une volonté de s'émanciper par la technologie, ou même plutôt d'une volonté d'émanciper la technologie.

En partant de la ville et du périphérique toulousain, la cabane lumineuse découvre ensuite ces autres lieux des Pyrénées audoises, jusqu'à cet arbre en haut d'une colline en côtoyant une diversité de faune et de flore. Les lumières saturées font donc l'expérience d'environnements plus calmes et qui ont d'autres logiques écosystémiques que celles des villes.

Et si les objets techniques pouvaient dialoguer avec les écosystèmes et s'intégrer respectueusement en leur sein?

Gilbert Simondon écrivait qu' " un objet technique peut être beau, lorsqu'il y a insertion dans le monde en un point et un moment remarquable. $\rangle^{5}$. Le technologique est bien aujourd'hui quelquechose de nomade, et si nous l'associons à la cabane comme figure artistique, c'est parce que la

\footnotetext{
${ }^{5}$ Gilbert Simondon, Du mode d'existence des objets techniques, Paris : éd. Aubier, 1989.
} 
cabane a pour nous quelque-chose de profondément utopique. Elle invite aux rêveries, à la construction de possibles à la fois ouverts et intimes, tout en restant accessible avec peu de moyens.

Si nous associons la cabane lumineuse à ces différents paysages, c'est bien pour éprouver ces contrastes entre la vitesse et la lenteur, mais aussi pour envisager d'associer les objets techniques à l'apaisement, à la pauvreté et à l'humilité. Et nous verrons même, en deuxième partie, comment nous avons cherché à donner un aspect sauvage à Luminale une fois qu'on la présenterait au public.

\section{Ecosystème, interactivité et cabane sauvage : de la scénographie paysagère à la scénographie d'installation interactive.}

En préambule de cette seconde partie, nous souhaiterions revenir sur la notion d'habitat énoncée en introduction. Nous postulions en effet qu'habiter c'est « faire système avec son environnement ». En effet, si travailler ensemble nous semblait si naturel, si nous avons toujours l'impression de parler le même langage artistique, c'est que nous appréhendons tous deux le monde au travers d'une certaine logique, qui se rapproche de la pensée systémique telle que définie par Edgar Morin. Nous percevons le monde comme un système, c'est à dire un ensemble complexe dont toutes les ramifications se tissent les unes aux autres. Habiter, c'est vivre pleinement ce jeu de relations, avec la distance et l'humilité nécessaire. C'est sur ce postulat que se base notre approche écosystémique.

Au travers de cette approche, nous avons arpenté différents environnements au contact desquels ont émergé Luminale. Cela nous a amené à faire évoluer Luminale d'une scénographie paysagère nomade à une scénographie d'installation interactive.

Le terme scénographie nous paraît tout à fait approprié ici pour désigner ces expériences menées dans ces différents environnements. Etymologiquement, la scénographie désigne littéralement l'écriture de l'espace. Ici l'espace est le paysage dans lequel le cabane s'inscrit, par un dialogue plastique, ainsi qu'en témoignent les différentes photographies. Michel Guérin, dans l'ouvrage Espaces Plastiques ${ }^{6}$, nous rappelle que le terme skéné, à l'origine du radical " scéno », désigne un espace tout particulier. La skéné est une tente qui se monte et se démonte au fil de déambulations, elle désignait particulièrement, dans le théâtre grec, la tente en arrière scène qui servait de coulisses. Le terme scénographie porte en lui les notions de l'éphémérité et du nomadisme. Lors de ses pérégrinations, Luminale est entrée en résonance avec son environnement, créant l'émergence, au sens systémique du terme, de scénographies paysagères.

Pensée dans cette complexité, elle n'est pas un objet plastique autonome, qui fonctionne en tant que tel par lui-même. C'est parce qu'elle est pensée ainsi comme scénographie, que Luminale prend sa dimension écosystémique. Chaque élément du paysage entre en interaction plastique avec elle. Cette expérience paysagère est au fondement de sa dimension installative. En effet, en arts plastiques, une installation désigne une œuvre tridimensionnelle qui implique une mise en espace, et dont tous les constituants fonctionnent dans leurs interrelations. En soi, une installation propose de fait une expérience immersive puisqu'elle implique une spectatorialité prise dans cet ensemble. Dans cette visée, toujours liée à la pensée complexe, il nous a paru tout naturel d'envisager une installation afin de transmuer cette scénographie paysagère dans un contexte d'exposition.

Dans un premier temps, nous avons souhaité recréer un environnement plastique qui rappelle le caractère sauvage de Luminale et sa fuite en marge des environnements urbains vers des endroits peu fréquentés.

\footnotetext{
${ }^{6}$ Michel Guérin, L'espace plastique, Bruxelles : éd. La Part de l'oeil, 2008, p. 24.
} 
Visuellement la terre au sol donne la sensation d'être face à une cabane au milieu des bois. L'aspect vieilli des matériaux, déjà évoqué plus haut, donne l'impression que notre cabane est là depuis longtemps, délaissée par l'humain.

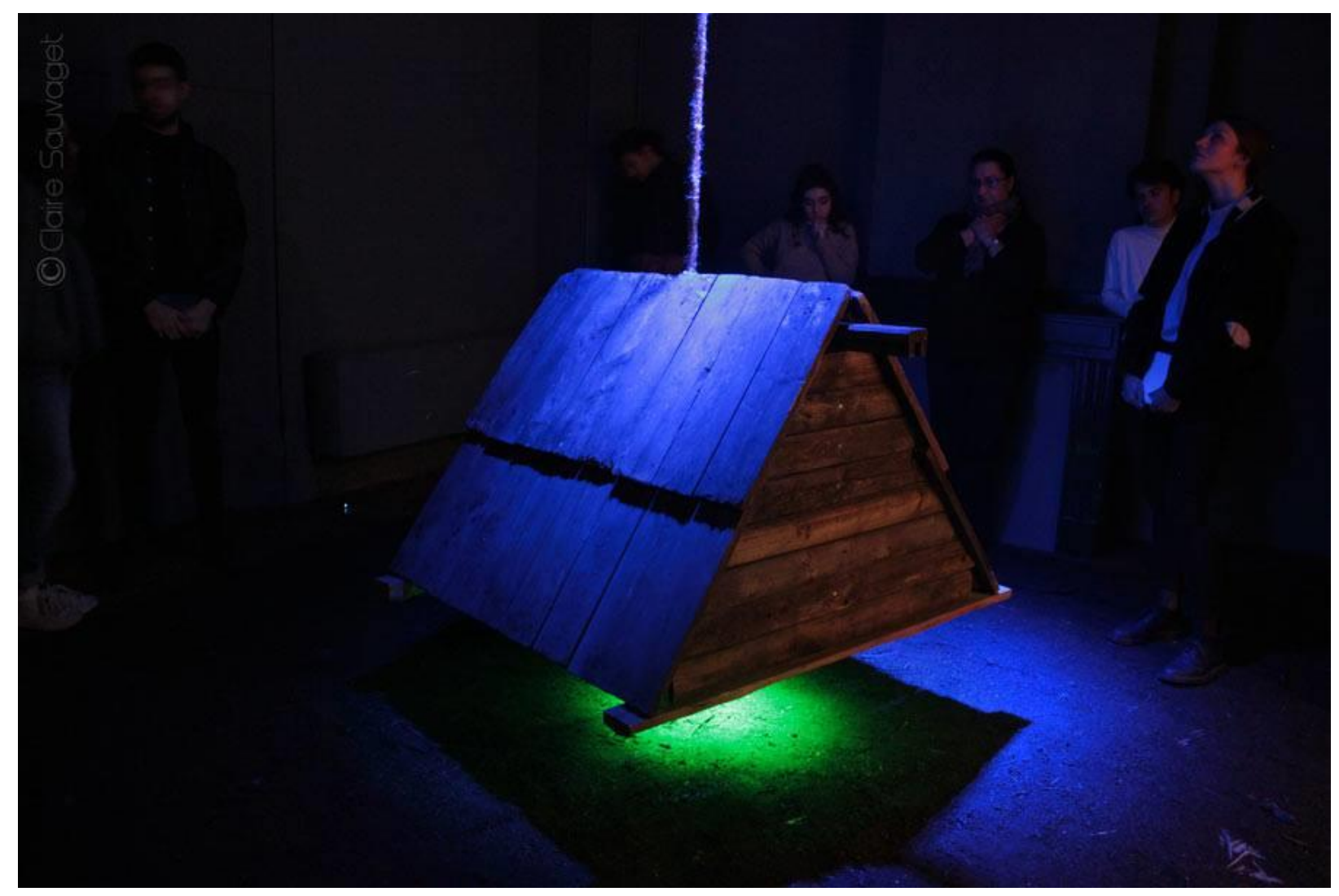

À partir des prises de sons effectuées dans les différents lieux d'accrochage, le travail de création sonore constitue un paysage sonore singulier. Aux alentours de la cabane, un système de diffusion donne à entendre des bruits paysagers reconnaissables. Du cœur de la cabane émanent des sons plus étranges. En dialogue avec la dimension visuelle de l'œuvre, la nature même de ces sons crée un espace plastique qui évoque cette dimension sauvage. Le faible éclairage ouvre un imaginaire nocturne. L'architecture du lieu d'exposition se fait oublier par la mise en lumière portée exclusivement au niveau de la cabane. Les sons dessinent alors un paysage hors champ.

La dimension lumineuse, évoquée plus haut, est en contraste avec les matériaux naturels patinés par le temps. Elle permet une défamiliarisation du spectateur avec l'objet cabane. Objet inconnu, elle l'invite ainsi à garder une certaine distance. Les sons étranges qui émanent de la cabane renforcent cette sensation. Les spectateurs et spectatrices savent intuitivement qu'ils doivent s'en approcher avec précaution. Ces éléments sonores et lumineux mis en espace évoluent au fil des actions spectatorielles par le biais d'un dispositif interactif. L'esthétique évoque le sauvage, mais cet aspect est avant tout caractérisé par le jeu d'interactions.

La dimension interactive ajoute des couches de complexité en tissant de nouveaux liens entre le spectateur et son environnement. Cela démultiplie la sensation d'interagir avec un être animal. En effet, elle est sensible au nombre de personnes, à leur proximité et à leurs mouvements. Lorsque des personnes s'approchent d'elle, la lumière et les sons que l'on perçoit à l'intérieur tendent à disparaître, comme si la cabane cherchait à échapper aux spectateurs et à se faire oublier.

Lorsque les personnes s'approchent de plus près, elles doivent se comporter avec douceur, sans faire de gestes brusques ou de déplacements trop francs. Elle semble alors s'éveiller. À l'intérieur, la 
lumière verte apparaît de nouveau et des sons étonnants, comme des étranges murmures, peuvent y être perçus.

Lorsque le nombre de personnes augmente, ou qu'elles s'éloignent de la cabane, cette relation intime s'efface. C'est là que les paysages sonores apparaissent aux alentours, évoquant différents environnements plus ou moins riches de sons. Plusieurs trames sonores paysagères sont lues en boucle de façon superposées.

Tout a été pensé pour donner l'impression au spectateurs et spectatrices qu'ils doivent apprivoiser cette cabane s'ils veulent observer ses subtilités sonores et lumineuses.

Captés lors des pérégrinations, les sons diffusés depuis l'intérieur de la cabane ont été pensés pour renforcer cette impression d'être en relation avec un être animal. Des transformations ont été opérées jusqu'à les rendre non-identifiables. Deleuze, dans l'analyse qu'il propose des peintures de Bacon, dans l'ouvrage Logique de la sensation ${ }^{7}$, propose la notion de figural, pour désigner ces figures qui, à la limite de l'abstraction, suggèrent des corps, suggèrent la chair, sans les représenter de façon explicite. C'est dans la logique de cette notion de figural que les sons ont été travaillés. Ainsi ils évoquent des phénomènes organiques sans pour autant les illustrer, laissant libre l'imaginaire des personnes qui les perçoivent. Contrairement aux sons paysagers qui sont diffusés sur des haut-parleurs classiques permettant une mise en espace, ces sons organiques sont diffusés par des transducteurs qui transmettent le son par contact avec la matière. Cachés sur les lattes de bois sec qui constituent la toiture, ces haut-parleurs transmettent leurs vibrations dans toute la cabane. Le son n'émane pas d'un point précis, il se répand. Si l'on effleure la cabane, on ressent nettement les vibrations du son. Elle semble non pas habitée, mais bien vivante. Cette expérience sensorielle tant auditive que tactile crée un lien d'intimité entre le spectateur et cette cabane.

Nous avons vu précédemment que la dimension poïétique de l'œuvre est liée à son caractère nomade. Née d'une expérience de déambulation, elle s'est construite sur la sensibilité aux paysages qui l'ont accueillie. Tous les environnements fréquentés par Luminale ont fait l'objet de captations sonores. Ces captations constituent une véritable mémoire de ces lieux. Cela alimente l'idée que l'œuvre est pensée comme un être vivant car elle porte alors en elle, de façon prégnante, cette multiplicité paysagère. Dans la définition même de la notion de paysage sonore, Murray Schaffer propose une contre-analogie avec la pratique photographique : «Un appareil photo permet en effet de saisir de façon immédiate et certaine les traits saillants d'un panorama visuel. Le micro n'opère pas de la même manière. Il recueille des détails, rapproche. ${ }^{8}$ Cela créé une relation proxime avec l'environnement sonore qui fait l'objet des captations. Luminale garde en mémoire ces sons, comme traces de ses déambulations. Au contact des spectateurs, elle retranscrit cette intimité vécue. Par ces rapprochements qui permettent la manifestation de détails, ne pourrions-nous pas alors envisager le paysage sonore, avec toute l'ambivalence que cela suggère, comme une approche portraitiste ? En effet, ce texte de Murray Schaffer peut permettre de poser un nouveau regard sur les photographies de Luminale. Ce sont des photographies de paysage qui n'ont pas de proportions panoramiques, même dans le plan le plus large, jusqu'à resserrer le cadrage à des plans moyens. Mais surtout, elles ont toutes été réalisées au format portrait. Sauvage, nomade, Luminale se présente comme un être vivant qui déambule, réagit aux présences humaines et porte la mémoire des lieux qu'elle a habité. Les photographies ne sont pas de simple témoignages ou photographies de paysage, si leur format a été intuitivement choisit dans sa relation formelle au sujet (la cabane suspendue, verticale), on peut les analyser comme portraits d'une entité paysagère.

\footnotetext{
${ }^{7}$ Gille Deleuze, Francis Bacon : Logique de la Sensation, Paris : Éd. de la Différence, 1996. p. 14

${ }^{8}$ Raymond Murray Schaffer, Le Paysage Sonore, Paris : éd. Jean-Claude Lattis, coll. Musique et Musiciens, 1979, p. 21.
} 
Dans l'œuvre interactive, le spectateur est dans l'habitat de l'œuvre, mis en présence de cette entité. Par les capteurs qui réagissent à sa présence, il est inclu dans le système technologique. Pour autant, fait-il véritablement système avec elle ? On va considérer que dans une relation œuvre/spectateur en général, et a fortiori dans une installation interactive, les liens qui se tissent sont suffisamment complexes pour conférer au spectateur une part d'auctorialité. Dans le cas de l'œuvre interactive en particulier, c'est par les actions attendues du spectateur, en fonction d'un programme informatique et d'un système technologique, que l'œuvre révèle ses formes en process. Le spectateur a alors un rôle à jouer dans la création et serait également auteur. Edmond Couchot parle d' « auteur aval ${ }^{9}$. Ici, si l'œuvre évolue au contact du spectateur, c'est qu'elle se laisse difficilement approcher. Appelant un certain comportement, l'œuvre conditionne l'expérience spectatorielle au point où le degré d'auctorialité est finalement assez réduit. Le spectateur n'est pas face à un panel de choix. Ses actions sont attendues. On peut se demander s'il y a véritablement une relation écosystémique. C'est dans une deuxième lecture que cette relation pourrait se créer. Habiter un espace, un environnement, c'est entrer dans une relation écosystémique en ayant pleine conscience des relations qui s'y tissent, et une pleine conscience de notre capacité à les infléchir.

Dans le comportement de la cabane, on retrouve une peur de l'agitation et de la vitesse. Elle nous incite alors à ralentir. Le spectateur habitera cette œuvre non pas en participant directement à sa dimension processuelle et jouant avec le système de captation. Il l'habitera comme on habite un paysage, en ralentissant, en prenant le temps de l'écouter. On peut y voir également une incitation à ralentir que ce soit dans nos modes de vie ou dans nos rapports au technologies. On peut alors se demander dans quelle mesure ces mêmes technologies infléchissent nos modes de vie. En étant calme, on peut poser un regarde poétique et contemplatif sur la manière dont les technologies habitent elles-mêmes nos environnements, et sur la façon dont on peut s'en saisir pour ouvrir de nouveaux champs de création.

\section{Conclusion}

Le phénomène qui se traduit à l'échelle du dispositif interactif a vocation à questionner notre rapport au monde. L'expérience spectatorielle vécue au contact de Luminale conduit à une certaine humilité. Elle amène à prendre conscience de notre imbrication dans un écosystème, et par là notre capacité à impacter tout autre élément par nos actions ou notre simple présence. Une première interprétation pourrait donc aller dans le sens d'une approche écologique dans l'acception d'une attention plus responsable portée à notre environnement. En questionnant le dispositif interactif qui dirige et infléchit les comportements des spectateurs, on touche au principal enjeu de ce travail. Il concerne les régimes de contraintes qui régissent nos vies et met en lumière les processus d'émancipation. Cette pensée peut être corroborée par une approche philosophique de la notion même de dispositif. Dans l'analyse de cette notion qu'il définit comme un « ensemble multilinéaire », Deleuze distingue des « lignes de pouvoir ${ }^{10}$, dont dépendent des « lignes de subjectivation ${ }^{11}$. Cela rejoint la pensée d'Agamben qui voit pour sa part dans les dispositifs des processus $\mathrm{d}^{\text {'assujettissement }}{ }^{12}$. Cette approche philosophique révèle ici la façon dont nous ouvrons des régimes d'individuation entre les lignes fortes qui caractérisent le dispositif technologique.

\footnotetext{
${ }^{9}$ Edmond Couchot, Des images, du temps, des machines, éd. Jacqueline Chambon, Nîmes, 2007, p.52.

${ }^{10}$ Gille Deleuze, «Qu'est-ce qu'un dispositif ? » in Deux Régimes de fous, Paris : éd. De Minuit, 2003, p. 319

11 Ibid.

${ }^{12}$ Giorgio Agamben, Qu'est-ce qu'un dispositif ?, Paris : éd. Payot et Rivage, 2007, p. 42 (coll. Rivage poche/ petite bibliothèque). 
L'idée de la cabane porte en elle ces processus d'émancipation, par les imaginaires qu'elle porte mais aussi par les actions concrètes qu'elle implique. Elle apparait alors paradigmatique de ces espaces d'individuation porteurs de singularité. En préambule de son ouvrage Cabanophiles, le charpentier et créateur de cabanes Yogan Samson-Bredel définit la cabane non pas par sa taille ou ses constituants architecturaux, mais par la philosophie qui a sous-tendu sa construction ${ }^{13}$. Cette philosophie, ce fil rouge qui relie les amateurs de cabane implique une prise de recul par rapport aux habitus du monde moderne dont elle va chercher les limites. Cela peut faire écho à la philosophie de Deleuze qui indique que «les lignes de subjectivation sont à l'extrême bord du dispositif ${ }^{14}$. La cabane est plus qu'un simple habitat, elle s'instaure comme une figure de l'habitat en nous invitant à fréquenter ces marges et en faire des espaces de liberté. De ce point de vue-là, on comprendra mieux comment les technologies deviennent vectrices de la dynamique émancipatrice paradigmatique de la cabane.

\section{Bibliographie}

AGAMBEN, Giorgio, Qu'est-ce qu'un dispositif ?, Paris : éd. Payot et Rivage, 2007 (coll. Rivage poche/ petite bibliothèque).

BACHELARD, Gaston, La poétique de l'espace, Paris, Presses universitaires de France, 2012.

COUCHOT, Edmond, Des images, du temps, des machines, Nîmes : éd. Jacqueline Chambon, 2007.

DELEUZE, Gille, Francis Bacon : Logique de la Sensation, Paris : Éd. de la Différence, 1996.

DELEUZE, Gille, «Qu'est-ce qu'un dispositif ? » in Deux Régimes de fous, Paris : éd. De Minuit, 2003.

GUERIN, Michel, L'espace plastique, Bruxelles : éd. La Part de l'oeil, 2008.

MURRAY SCHAFFER, Raymond, Le Paysage Sonore, Paris : éd. Jean-Claude Lattis, coll. Musique et Musiciens, 1979.

SAMSON-BREDEL, Yogan, Cabanophiles, Cabanistan : éd. La cabane d'édition, 2017.

SIMONDON, Gilbert, Du mode d'existence des objets techniques, Paris : éd. Aubier, 1989.

THOREAU, Henry David, Walden ou La vie dans les bois, Paris: éd. Climats, 1967, [traduction et notes par Germaine Landré-Augier].

\footnotetext{
${ }^{13}$ Yogan Samson-Bredel, Cabanophiles, éd. La cabane d'édition, Cabanistan, 2017

${ }^{14}$ Gille Deleuze, Op. Cit.
} 